\title{
Phenolic compounds from Pamotrema dilatatum growing in Lam Dong province
}

\section{Duong Thuc Huy}

Department of Chemistry, Ho Chi Minh City University of Education, $280 \mathrm{An}$ Duong Vuong Street, District 5, Ho Chi Minh City, Vietnam

Email: thuchuy84@yahoo.com

\section{History}

- Received: 2018-11-12

- Accepted: 2019-01-04

- Published: 2019-01-26

DOI :

https://doi.org/10.32508/stdj.v22i1.1010

\section{Check for updates}

\section{Copyright}

(c) VNU-HCM Press. This is an openaccess article distributed under the terms of the Creative Commons Attribution 4.0 International license.

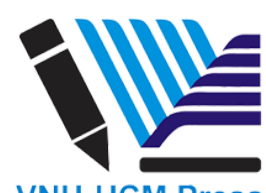

\begin{abstract}
Introduction: Only chemical study on the lichen Parmotrema dilatatum was found so far in the world. The lichen Parmotrema dilatatum widely distributed in Lam Dong province, Vietnam has been studied about the isolation and elucidation of several metabolites.

Methods: Phytochemical study on the polar fractions of this lichen was carried out by using various chromatographic methods including thin-layer chromatography and normal phase silica gel chromatography.

Results: Eight phenolic compounds were isolated. The chemical structures of isolated compounds were unambiguously elucidated by NMR spectroscopy and comparison with the data in the literature. These are 8'-O-methylsalazinic acid, salazinic acid, 8'-O-methylprotocetraric acid, diffractaic acid, lecanorin, lecanoric acid, isolecanoric acid, and diorcinolic acid.

Conclusion: Among them, 8'-O-methylsalazinic acid was found as a new natural product.

Key words: depside, depsidone, diphenyl etther, Lichen, Pamotrema dilatatum, phenolic compound
\end{abstract}

\section{INTRODUCTION}

Lichen metabolites endowed with various bioactivities, especially phenolic compounds such as depsides, depsidones and diphenyl ethers ${ }^{1}$. Depsidones showed antiviral, antibacterial, and enzyme inhibitory activities while depsides and diphenyl ethers exhibited strong cytotoxicity toward many cancer cell lines and other potent bioactivities ${ }^{2}$.

Vietnamese lichens have much attracted chemists to investigate new metabolites. The Parmotrema genera popularly growing in Vietnam, i.e P. tsavoense, $P$. sancti-angelii, and $P$. prasorediosum produced many new and novel compounds having interesting biological activities ${ }^{3-5}$. In the course of our systematic research on lichen substances from the Vietnamese lichens, we have examined Parmotrema dilatatum, widely distributed in the high attitude in South Vietnam. Chemical data on Parmotrema dilatatum are scarce with only report of isolation of two major components, salazinic acid and atranorin ${ }^{6}$. Herein describes the isolation of eight compounds from the polar extract of the lichen Parmotrema dilatatum. Their chemical structures were defined by NMR and MS spectroscopy in accordance with the data reported in the literature.

\section{METHODS}

\section{General experimental procedures}

Bruker Advance III $\left(500 \mathrm{MHz}\right.$ for ${ }^{1} \mathrm{H}$ NMR and $125 \mathrm{MHz}$ for ${ }^{13} \mathrm{C}$ NMR) spectrometer with TMS as internal standard recorded NMR spectra. Chemical shifts are expressed in ppm with reference of acetone- $d_{6}$ at $\delta_{H} 2.05, \mathrm{~d}_{C} 206.26$ and 29.84 and of dimethylsulfoxide- $d_{6}$ at $\delta_{H} 2.50$ and $\delta_{C}$ 39.52. The HR-ESI-MS were recorded on a HR-ESI-MS Bruker microOTOF Q-II. TLC was carried out on precoated silica gel $60 \mathrm{~F}_{254}$ or silica gel $60 \mathrm{RP}-18 \mathrm{~F}_{254} \mathrm{~S}$ (Merck Millipore, Billerica, Massachusetts, USA) and spots were visualized by spraying with $10 \% \mathrm{H}_{2} \mathrm{SO}_{4}$ solution followed by heating. Gravity column chromatography was performed with silica gel $60(0.040-0.063 \mathrm{~mm})$ (HiMedia, Mumbai, India).

\section{Plant material}

Lichen thalli were separated from rocks in Lam Dong province, Vietnam in August-September 2015. The scientific name was defined by Dr. Wetchasart Polyiam, Lichen Research Unit, Department of Biology, Faculty of Science, Ramkhamhaeng University. A voucher specimen (No UP-002) was deposited in the herbarium of the Department of Organic Chemistry, University of Education - Ho Chi Minh City Vietnam. 


\section{Extraction and isolation}

The thallus material was washed under a flow of tap water, prior to being rinsed with distilled water. The lichen was air-dried at ambient temperature $\left(35^{\circ} \mathrm{C}\right)$ to avoid thermal decomposition. Clean, ground and dry material $(703 \mathrm{~g})$ was macerated in acetone $(3 \times 10$ L) at ambient temperature, and the filtrated solution was concentrated in vacuo to dryness to afford a crude acetone extract (112.54 g). This crude extract was subjected to normal phase silica gel quick column chromatography, eluted consecutively with $n$ hexane, dichloromethane, ethyl acetate, acetone, and methanol to afford five extracts PH (3.02 g), PC (6.17 g), PEA (44.53 g), PA (31.71 g), and PM (4.91 g), respectively.

The extract PA was washed three times by the mixture of acetone-dichloromethane (1:1) to afford the precipitate PAT $(22.0 \mathrm{~g})$ and the solution PAS (9.71 g). The solution PAS was concentrated and chromatographed by CC, eluted with the solvent system $n$-hexane-dichloromethane-ethyl acetate-acetone-acetic acid (350:100:40:25:10) to yield six fractions, PAS1-PAS6. Fraction PAS1 (1.5 g) was fractionated by CC, eluted with the same solvent system to afford three sub-fractions PAS1.1-3. Fraction PAS1.1 (251 mg) was rechromatographed to afford two compounds $3(2.3 \mathrm{mg})$ and $\mathbf{4}(2.3 \mathrm{mg})$. Fraction PAS4 (3.3 g) was fractionated by CC, eluted with the solvent system $n$-hexane-dichloromethane-ethyl acetate-acetone-acetic acid (175:100:40:25:10) to afford three subfractions PAS4.1-3. Purifying the subfraction PAS4.2 (700 mg) resulted in two compounds 1 (31 mg) and 2 (430 mg) Figure 1.

The extract PEA (44.53 g) was applied to CC, eluted with $n$-hexane-ethyl acetate-acetone (4:1:1) to afford ten fraction PEA1-10. Fraction PEA1 (4.1 g) was fractionated by CC, eluted with the solvent system n-hexane-dichloromethane-ethyl acetate-acetoneacetic acid (350:100:40:25:10) to afford three fractions PEA1.1-3. Fraction PEA1.3 (680 mg) was applied to preparative TLC, eluted with the previously described solvent system to afford four compounds $5(8.7 \mathrm{mg})$, 6 (102 mg), 7 (6.3 mg), and 8 (2.3 mg) Figure 1.

- 8'-O-methylsalazinic acid (1). White amorphous powder; HR-ESI-MS $m / z 401.05500$ [M-H $^{-}$(calcd for $\mathrm{C}_{19} \mathrm{H}_{14} \mathrm{O}_{10}-\mathrm{H}, 401.05087$ ); the ${ }^{1} \mathrm{H}$ and ${ }^{13} \mathrm{C} \mathrm{NMR}$ (DMSO-d6) spectroscopic data, see Table 1.

- Salazinic acid (2). White amorphous powder; the ${ }^{1} \mathrm{H}$ and ${ }^{13} \mathrm{C}$ NMR (DMSO-d6) spectroscopic data, see Table 1.

- 8'-O-methylprotocetraric acid (3). White amorphous powder; the ${ }^{1} \mathrm{H}$ and ${ }^{13} \mathrm{C}$ NMR (DMSO-d6) spectroscopic data, see Table 1.
- Diffractaic acid (4). White amorphous powder; the ${ }^{1} \mathrm{H}$ and ${ }^{13} \mathrm{C}$ NMR (DMSO-d6) spectroscopic data, see Table 2.

- Lecanorin (5). White amorphous powder; the ${ }^{1} \mathrm{H}$ and ${ }^{13} \mathrm{C}$ NMR (Acetone-d6) spectroscopic data, see Table 2.

- Lecanoric acid (6). White amorphous powder; the ${ }^{1} \mathrm{H}$ and ${ }^{13} \mathrm{C}$ NMR (Acetone-d6) spectroscopic data, see Table 2.

- Isolecanoric acid (7). White amorphous powder; the ${ }^{1} \mathrm{H}$ and ${ }^{13} \mathrm{C}$ NMR (Acetone-d6) spectroscopic data, see Table 2.

- Diorcinolic acid (8). White amorphous powder; the ${ }^{1} \mathrm{H}$ and ${ }^{13} \mathrm{C}$ NMR (Acetone-d6) spectroscopic data, see Table 2.

\section{RESULTS AND DISCUSSION}

Compound 1 was obtained as white amorphous powder with the molecular formula was determined by HR-ESI-MS data as $\mathrm{C}_{19} \mathrm{H}_{14} \mathrm{O}_{10}$. The ${ }^{1} \mathrm{H}$ NMR spectra of 1 showed the presence of one formyl $\left(\delta_{H} 10.60\right.$, $1 \mathrm{H}, \mathrm{s})$, one aromatic proton $\left(\delta_{H} 6.84,1 \mathrm{H}, \mathrm{s}\right)$, one methine proton $\left(\delta_{H} 6.62,1 \mathrm{H}, \mathrm{s}\right)$, one oxygenated methylene proton $\left(\delta_{H} 4.53,2 \mathrm{H}, \mathrm{s}\right)$, one methoxy group $\left(\delta_{H} 3.26,3 \mathrm{H}, \mathrm{s}\right)$, one methyl groups $\left(\delta_{H} 2.55,3 \mathrm{H}\right.$, s). The ${ }^{13} \mathrm{C}$ NMR spectra showed the presence of one aldehyde carbon $\left(\delta_{C} 193.1\right)$, two carboxy carbon $\left(\delta_{C} 166.0\right.$ and 161.5), twelve aromatic carbons in the zone $\delta_{C}$ 100.0-166.0, one hemiacetal carbon $\left(\delta_{C}\right.$ 95.1), two oxygenated carbons ( $\delta_{C} 62.0$ and 57.3), and one methyl carbon $\left(\delta_{C} 21.0\right)$. The HMBC correlation of the methoxy group at $\delta_{H} 3.26\left(\delta_{C} 57.3\right)$ with carbon C- 8 ' indicated the presence of the methoxy group at this carbon. The comparison NMR spectral data of 1 have with those of 8'-O-methylsalazinic acid Elix et al. (1999) showed that they were identical. Thus, 1 was elucidated as 8'-O-methylsalazinic acid ${ }^{7}$ Figure 2. Compound 2 was obtained as white amorphous powder. The ${ }^{1} \mathrm{H}$ NMR and HSQC spectra of $\mathbf{2}$ showed the presence of one chelated hydroxyl group $\delta_{H}$.

Compound 3 was obtained as white amorphous powder. The ${ }^{1} \mathrm{H}$ NMR and HSQC spectra of $\mathbf{3}$ showed the presence of one formyl $\left(\delta_{H}\right.$.

Compound 4 was isolated as obtained as colorless needle. The ${ }^{1} \mathrm{H}$ NMR spectra of $\mathbf{4}$ showed the presence of one two aromatic protons $\left(\delta_{H}\right.$ was elucidated as diffractaic acid.

Compound 5 was obtained as white amorphous powder. The ${ }^{1} \mathrm{H}$ NMR spectra of $\mathbf{5}$ indicated the presence of one chelated hydroxy group $\left(\delta_{H}\right.$.

Compound 6 was obtained as white amorphous powder. NMR data of $\mathbf{6}$ was highly similar with those of 
<smiles></smiles>

$1 \mathrm{R}=\mathrm{OMe}$ $2 \mathrm{R}=\mathrm{OH}$<smiles>[R]c1c(C)cc(OC(=O)c2c(C)cc(O)cc2O)cc1O</smiles><smiles>COc1c(O)c(C(=O)O)c(C)c2c1OC(=O)c1c(C)cc(O)c(C=O)c1O2</smiles><smiles>Cc1cc(O)cc(O)c1C(=O)Oc1cc(O)cc(Oc2cc(O)cc(O)c2C(=O)O)c1C(=O)O</smiles>

Figure 1: Chemical structures of 8'-O-methylsalazinic acid (1), salazinic acid (2), 8'-O-methylprotocetraric acid (3), diffactaic acid (4), lecanorin (5), lecanoric acid (6), isolecanoric acid (7), and diorcinolic acid (8).

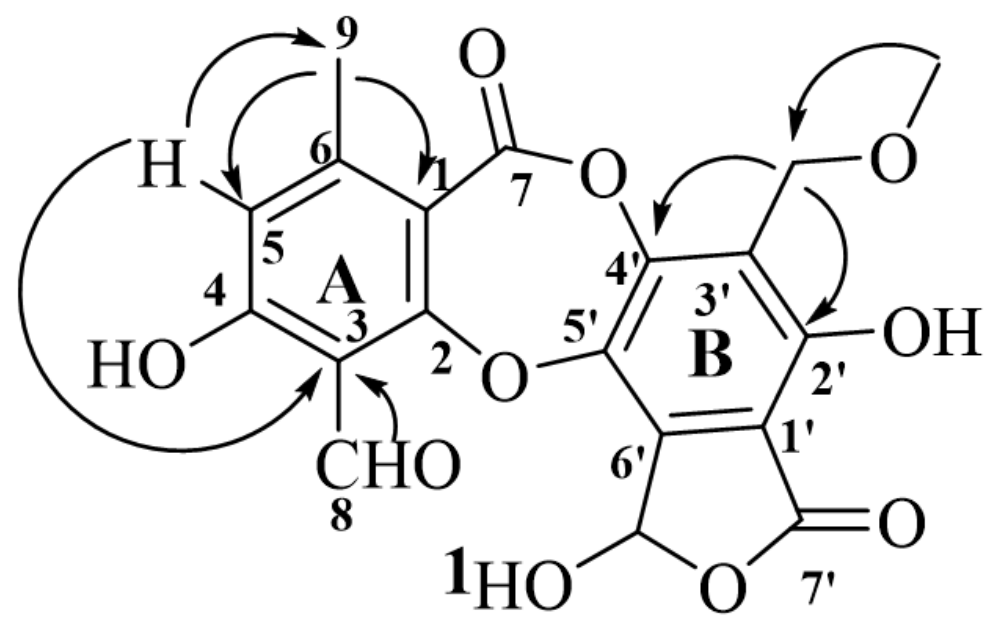

Figure 2: Key Heteronuclear Multiple Bond Correlations of 1.

$\mathbf{5}$, except for the absence of one aromatic proton in the B-ring $\left(\delta_{H}\right.$ was identified as lecanoric acid.

Compound 7 was obtained as white amorphous powder. The ${ }^{1} \mathrm{H}$ NMR spectra showed the presence of one chelated hydroxy group $\left(\delta_{H} 11.311 \mathrm{H}, s\right)$, four aromatic protons $\left(\delta_{H} 6.60,1 \mathrm{H}, s ; 6.52,1 \mathrm{H}, s ; 6.40,1 \mathrm{H}, s\right.$; $6.32,1 \mathrm{H}, s)$, and two methyl protons $\left(\delta_{H} 2.66 ; 3 \mathrm{H}, s\right.$ and $2.61,3 \mathrm{H}, s)$. NMR data of the A-ring of 7 was highly similar with those of 7 , indicating that they share the same A-ring. The differences were the signals of the B-ring, including the upfield chemical shift of C-4' $\left(\delta_{C}\right.$ was elucidated as isolecanoric acid.

Compound 8 was obtained as white amorphous powder. The ${ }^{1} \mathrm{H}$ NMR spectra showed the presence of one chelated hydroxy group $\left(\delta_{H} 11.33,1 \mathrm{H}, \mathrm{s}\right)$, four aromatic methines $\left(\delta_{H} 6.53,1 \mathrm{H}, d, 2.0 \mathrm{~Hz} ; 6.44,1 \mathrm{H}, d\right.$,
$2.0 \mathrm{~Hz} ; 6.38,1 \mathrm{H}, d, 2.5 \mathrm{~Hz} ; 6.29,1 \mathrm{H}, d, 2.5 \mathrm{~Hz}$ ), and two methyls $\left(\delta_{H} 2.65,3 \mathrm{H}\right.$, s and $\left.2.59,3 \mathrm{H}, \mathrm{s}\right)$. The ${ }^{13} \mathrm{C}$ NMR spectrum confirmed the presence of fifteen carbons comprising two carboxyl carbons $\left(\delta_{C}\right.$ was elucidated as diorcinolic acid.

Salazinic acid is a representive for a rare depsidone class having a hydroxymethylene moiety in the Bring with four compounds reported so far. Salazinic acid was isolated as a major component of the studied lichen. 8'-O-Methylsalazinic acid was a methylated derived from salazinic acid. Elix and coworkers (1999) converted quaesitic acid (9) to 8'-Omethylsalazinic acid (1) when storing quaesitic acid in methanol at ambient temperature. The transesterification occurred at C-8' of quaesitic acid led 
Table 1: Nuclear magnetic resonance of compounds 1 - 3 (in DMSO-d6)

\begin{tabular}{|c|c|c|c|c|c|c|}
\hline & \multicolumn{2}{|c|}{1} & \multicolumn{2}{|l|}{2} & \multicolumn{2}{|c|}{3} \\
\hline & $\begin{array}{c}\delta \mathbf{H}, \\
\mathbf{J}(\mathbf{H z})\end{array}$ & $\delta \mathbf{C}$ & $\begin{array}{c}\delta \mathbf{H}, \\
\mathbf{J}(\mathbf{H z})\end{array}$ & $\delta \mathbf{C}$ & $\begin{array}{c}\delta \mathbf{H}, \\
\mathrm{J}(\mathbf{H z})\end{array}$ & $\delta \mathbf{C}$ \\
\hline 1 & & 111.9 & & 112.2 & & 111.8 \\
\hline 2 & & 161.6 & & 160.0 & & 164.4 \\
\hline 3 & & nd & & nd & & 112.3 \\
\hline 4 & & 165.7 & & 164.0 & & 163.8 \\
\hline 5 & $6.84, \mathrm{~s}$ & 117.9 & $6.88, \mathrm{~s}$ & 117.9 & $6.78, \mathrm{~s}$ & 116.9 \\
\hline 6 & & 152.0 & & 152.0 & & 151.8 \\
\hline 7 & & 161.5 & & 160.3 & & 161.3 \\
\hline 8 & $10.60, \mathrm{~s}$ & 193.1 & $10.45, \mathrm{~s}$ & 193.1 & $10.54, \mathrm{~s}$ & 191.8 \\
\hline 9 & $2.55, \mathrm{~s}$ & 21.0 & $2.45, \mathrm{~s}$ & 21.4 & $2.45, \mathrm{~s}$ & 21.3 \\
\hline 4- $\mathrm{OH}$ & & & $12.06, \mathrm{~s}$ & & & \\
\hline $1^{\prime}$ & & 110.8 & & 110.6 & & 111.5 \\
\hline $2^{\prime}$ & & 152.1 & & 152.1 & & 158.2 \\
\hline $3^{\prime}$ & & 125.0 & & 123.8 & & 115.1 \\
\hline $4^{\prime}$ & & 145.1 & & 145.1 & & 145.1 \\
\hline 5 & & 144.7 & & 144.7 & & 141.2 \\
\hline 6 & & nd & & 137.6 & & 131.2 \\
\hline 7 & & 166.0 & & 165.0 & & 170.4 \\
\hline & & 62.0 & $4.64, \mathrm{~s}$ & 52.8 & $4.43, \mathrm{~s}$ & 62.4 \\
\hline $8^{\prime}$ & $4.53, \mathrm{~s}$ & & & & & \\
\hline $9^{\prime}$ & $6.62, \mathrm{br}$ & 95.1 & $6.80, \mathrm{~s}$ & 95.0 & $2.34, \mathrm{~s}$ & 14.4 \\
\hline $\mathrm{OCH}_{3}$ & $3.26, \mathrm{~s}$ & 57.3 & & & $3.19, \mathrm{~s}$ & 57.3 \\
\hline
\end{tabular}

nd: not determined

to the formation of $8^{\prime}-O$-methylsalazinic acid (1) and furmaric acid (10) (seeFigure 3 ). In our case, the solvent methanol has not been used during experimental process thus such esterification or etherification would not happen. At this scenario, we proposed that compound $\mathbf{1}$ was isolated as a new natural product.

Honda and coworkers (1999) previously studied this lichen with the isolation and identification of two compounds atranorin and salazinic acid ${ }^{6}$. Thus, compounds 3-7 were reported in the first time from the lichen Parmotrema dilatatum. Orcinol-derieved diphenyl ethers which have the similar skeleton as compound $\mathbf{8}$ are quite common from the fungus source $^{8}$. Nevertheless, diphenyl ethers seldom encountered from the Parmotrema genera ${ }^{9}$. This is the first time diphenyl ether diorcinolic acid (8) found in the Parmotrema lichens.

\section{CONCLUSIONS}

From Pamotrema dilatatum collected in Lam Dong province, eight phenolic compounds were isolated and elucidated, including 8'-O-methylsalazinic acid (1), salazinic acid (2), 8'-O-methylprotocetraric acid (3), diffactaic acid (4), lecanorin (5), lecanoric acid 
Table 2: Nuclear magnetic resonance data of compounds 4-8*

\begin{tabular}{|c|c|c|c|c|c|c|c|c|c|c|}
\hline Positi & $4^{a}$ & & $5^{b}$ & & $6^{b}$ & & $7^{b}$ & & $\mathbf{8}^{b}$ & \\
\hline & $\begin{array}{l}\delta \mathbf{H}, \\
\mathbf{J}(\mathbf{H z})\end{array}$ & $\delta \mathbf{C}$ & $\begin{array}{l}\delta \mathbf{H}, \\
\mathbf{J}(\mathbf{H z})\end{array}$ & $\delta \mathbf{C}$ & $\begin{array}{l}\delta \mathbf{H} \\
\mathbf{J}(\mathbf{H z})\end{array}$ & $\delta \mathbf{C}$ & $\begin{array}{l}\delta \mathbf{H} \\
\mathbf{J}(\mathbf{H z})\end{array}$ & $\delta \mathbf{C}$ & $\begin{array}{l}\delta \mathbf{H}, \\
\mathbf{J}(\mathbf{H z})\end{array}$ & $\delta \mathbf{C}$ \\
\hline 1 & & 119.3 & & 110.6 & & 105.5 & & 103.9 & & 104.1 \\
\hline 2 & & 156.4 & & 164.2 & & 162.2 & & 163.4 & & 165.7 \\
\hline 3 & & 116.4 & $\begin{array}{l}6.28, \mathrm{~d}, \\
2.5\end{array}$ & 101.9 & $\begin{array}{l}6.28, \mathrm{~d}, \\
2.5\end{array}$ & 100.8 & 6.32 , brs & 100.9 & $\begin{array}{l}6.29 \\
\mathrm{~d}, \\
2.5\end{array}$ & 100.8 \\
\hline 4 & & 161.3 & & 159.1 & & 162.7 & & 165.1 & & 163.0 \\
\hline 5 & $6.45, \mathrm{~s}$ & 108.5 & $\begin{array}{l}6.37, \mathrm{~d}, \\
2.5\end{array}$ & 107.4 & $\begin{array}{l}6.37, \mathrm{~d}, \\
2.5\end{array}$ & 111.8 & 6.40 , brs & 107.6 & $\begin{array}{l}6.38 \\
\mathrm{~d}, \\
2.5\end{array}$ & 111.8 \\
\hline 6 & & 134.8 & & 144.7 & & 141.6 & & 143.8 & & 143.8 \\
\hline 7 & & 165.5 & & 174.4 & & 167.9 & & 165.9 & & 169.8 \\
\hline 8 & $2.23, \mathrm{~s}$ & 19.5 & $2.59, \mathrm{~s}$ & 24.4 & $2.61, \mathrm{~s}$ & 22.5 & $2.61, \mathrm{~s}$ & 23.7 & $\begin{array}{l}2.59, \\
\mathrm{~s}\end{array}$ & 23.6 \\
\hline 9 & $1.90, \mathrm{~s}$ & 8.7 & & & & & & & & \\
\hline $1^{\prime}$ & & 111.5 & $\begin{array}{l}6.62, \mathrm{t}, \\
2.0\end{array}$ & 114.7 & & 116.2 & & 115.1 & & 116.1 \\
\hline $2^{\prime}$ & & 159.5 & & 154.5 & & 155.9 & & 152.2 & & 164.4 \\
\hline $3^{\prime}$ & & 116.0 & $\begin{array}{l}6.57, \mathrm{~d}, \\
2.0\end{array}$ & 114.5 & $\begin{array}{l}6.73, \mathrm{~d}, \\
2.5\end{array}$ & 111.0 & 6.52 , brs & 107.6 & $\begin{array}{l}6.53 \\
\mathrm{~d}, \\
2.0\end{array}$ & 107.3 \\
\hline $4^{\prime}$ & & 152.2 & & 152.0 & & 151.9 & & 163.4 & & 151.6 \\
\hline 5 & $6.62, \mathrm{~s}$ & 115.7 & $\begin{array}{l}6.57, \mathrm{~d}, \\
2.0\end{array}$ & 112.9 & $\begin{array}{l}6.76, \mathrm{~d}, \\
2.5\end{array}$ & 116.9 & 6.60 , brs & 114.3 & $\begin{array}{l}6.44 \\
\mathrm{~d}, \\
2.0\end{array}$ & 114.1 \\
\hline $6^{\prime}$ & & 139.0 & & 141.1 & & 137.9 & & 143.5 & & 143.5 \\
\hline $7^{\prime}$ & & 173.1 & & & & 171.2 & & 169.9 & & 174.7 \\
\hline $8^{\prime}$ & $2.34, \mathrm{~s}$ & 22.8 & $2.29, \mathrm{~s}$ & 21.4 & $2.65, \mathrm{~s}$ & 21.5 & $2.66, \mathrm{~s}$ & 23.0 & $\begin{array}{l}2.65, \\
s\end{array}$ & 22.8 \\
\hline $9^{\prime}$ & $1.98, \mathrm{~s}$ & 8.9 & & & & & & & & \\
\hline $\begin{array}{l}2- \\
\mathrm{OMe}\end{array}$ & $3.68, \mathrm{~s}$ & 61.8 & & & & & & & & \\
\hline $\begin{array}{l}4- \\
\mathrm{OMe}\end{array}$ & $3.60, \mathrm{~s}$ & 55.8 & & & & & & & & \\
\hline $\begin{array}{l}2- \\
\mathrm{OH}\end{array}$ & & & $11.30, \mathrm{~s}$ & & & & & & & \\
\hline $\begin{array}{l}2^{\prime}- \\
\mathrm{OH}\end{array}$ & & & & & & & $11.31, \mathrm{~s}$ & & $\begin{array}{l}11.33, \\
\mathrm{~s}\end{array}$ & \\
\hline
\end{tabular}


<smiles>COOC(=O)C=CC(=O)OCc1c(O)c2c(c3c1OC(=O)c1c(C)cc(O)c(C=O)c1O3)C(O)OC2=O</smiles>

Quaesitic acid (9)<smiles>COCc1c([OH2+])c2c(c3c1OC(=O)c1c(C)cc(O)c(C=O)c1O3)C(O)OC2=O</smiles>

8 -O-Methylsalazinic acid (1)

Furmaric acid (10)
(6), isolecanoric acid (7), and diorcinolic acid (8). Compound 1 was reported as a new natural product while other compounds 3-8 were found in the studied lichen for this first time.

\section{ABBREVIATIONS}

${ }^{13}$ C NMR: Carbon-13 nuclear magnetic resonance

${ }^{1}$ H NMR: Proton nuclear magnetic resonance

brs: broad singlet

CC: column chromatography

d: doublet

DMSO: Dimethyl sulfoxide $\left(\mathrm{CD}_{3} \mathrm{SOCD}_{3}\right)$

HMBC: Heteronuclear multiple bond correlation

HPLC: High-performance liquid chromatography

HSQC: Heteronuclear single quantum coherence

s: singlet

TLC: Thin layer chromatography

\section{COMPETING INTERESTS}

The authors declare no competing financial interest.

\section{AUTHORS' CONTRIBUTIONS}

Duong T. H. has contributed in conducting experiments, acquisition of data, interpretation of data, searching the bibliography and gave final approval of the manuscript to be submitted.

\section{ACKNOWLEDGMENTS}

We would like to thank Dr. Wetchasart Polyiam for lichen identification.

\section{REFERENCES}

1. Brandão LF, Alcantara GB, Matos MF, Bogo D, Freitas DS, Oyama NM. Cytotoxic evaluation of phenolic compounds from lichens against melanoma cells. Chemical \& Pharmaceutical Bulletin. 2013;61:176-83. Available from: DOI:10.1248/cpb.c12-00739.

2. Müller K. Pharmaceutically relevant metabolites from lichens. Applied Microbiology and Biotechnology. 2001;56(1-2):9-16.

3. Huynh BL, Duong TH, Do TM, Pinnock TG, Pratt LM, Yamamoto S. New [gamma]-Lactone carboxylic Acids from the Lichen Parmotrema praesorediosum (Nyl.) Hale, Parmeliaceae. Records of Natural Products. 2016;10:332-40.

4. Duong TH, Huynh BL, Chavasiri W, Chollet-Krugler M, Nguyen VK, Nguyen TH. New erythritol derivatives from the fertile form of Roccella montagnei. Phytochemistry. 2017;137:15664. Available from: DOI:10.1016/j.phytochem.2017.02.012.

5. Duong TH, Ha XP, Chavasiri W, Beniddir MA, Genta-Jouve G, Boustie J. Sanctis A-C: Three Racemic Procyanidin Analogues from the Lichen Parmotrema Sancti-Angelii. European Journal of Organic Chemistry. 2018;2018:2247-53. Available from: DOI:10.1002/ejoc.201800202.

6. Honda NK, Pavan FR, Coelho RG, de Andrade Leite SR, Micheletti AC, Lopes TI. Antimycobacterial activity of lichen substances. Phytomedicine. 2010;17:328-32. Available from: DOI:10.1016/j.phymed.2009.07.018.

7. Elix JA, Wardlaw JH. The Structure of Chalybaeizanic Acid and Quaesitic Acid, Two New Lichen Depsidones Related to Salazinic Acid. Australian Journal of Chemistry. 1999;52:7135. Available from: Doi:10.1071/ch99056.

8. Liu S, Wang H, Su M, Hwang GJ, Hong J, Jung JH. New metabolites from the sponge-derived fungus Aspergillus sydowii J05B7F-4. Natural Product Research. 2017;31:1682-6. Available from: Doi:10.1080/14786419.2017.1289205.

9. Duong TH, Chavasiri W, Boustie J, Nguyen KP. New MetaDepsidones and Diphenyl Ethers from the Lichen Parmotrema Tsavoense (Krog \& Swinscow) Krog \& Swinscow, Parmeliaceae. Tetrahedron. 2015;71:9684-91. Available from: DOI:10.1016/j. tet.2015.06.107. 